\title{
Crystal structures of wild-type and mutated cyclophilin B that causes hyperelastosis cutis in the American quarter horse
}

\author{
Sergei P Boudko ${ }^{1,2}$, Yoshihiro Ishikawa ${ }^{1,2}$, Thomas F Lerch², Jay Nix ${ }^{3}$, Michael S Chapman ${ }^{2}$ \\ and Hans Peter Bächinger ${ }^{1,2^{*}}$
}

\begin{abstract}
Background: Hyperelastosis cutis is an inherited autosomal recessive connective tissue disorder. Affected horses are characterized by hyperextensible skin, scarring, and severe lesions along the back. The disorder is caused by a mutation in cyclophilin B.

Results: The crystal structures of both wild-type and mutated (Gly6->Arg) horse cyclophilin B are presented. The mutation neither affects the overall fold of the enzyme nor impairs the catalytic site structure. Instead, it locally rearranges the flexible $\mathrm{N}$-terminal end of the polypeptide chain and also makes it more rigid.
\end{abstract}

Conclusions: Interactions of the mutated cyclophilin B with a set of endoplasmic reticulum-resident proteins must be affected.

Keywords: Peptidyl prolyl cis-trans-isomerase (PPlase), Cyclophilin B (CypB), Endoplasmic reticulum, Chaperone, Protein complex, Calreticulin, P-domain, Lysyl hydroxylase, Collagen, HERDA

\section{Background}

Hyperelastosis cutis $(\mathrm{HC})$ or Hereditary equine regional dermal asthenia (HERDA), a degenerative skin disease in Quarter horses has been reported since 1978, in which the skin is described as hyperelastic, fragile and thin, with slow-healing wounds characterized by atrophic scars [1]. Lesions may be single or multiple, and are most commonly seen on the dorsum, although they may also occur on the legs [1]. Foals affected with HC rarely show symptoms at birth, but develop seromas, hematomas, and ulcerations primarily along the dorsal aspect that progressively worsen in frequency and severity with age. Many cases are not identified until the horses have a saddle on their backs, and lesions are most commonly found along the dorsal aspect, coincident with where the saddle would rest. The majority of affected horses are

\footnotetext{
* Correspondence: hpb@shcc.org

${ }^{1}$ Research Department, Shriners Hospital for Children, Portland, OR 97239, USA

${ }^{2}$ Department of Biochemistry and Molecular Biology, Oregon Health \& Science University, Portland, OR 97239, USA

Full list of author information is available at the end of the article
}

euthanized. Published case reports describing HC have compared the disease to Ehlers-Danlos syndrome [2].

A missense mutation in cyclophilin B (CypB) has been identified and pointed to a causal candidate gene for $\mathrm{HC}$ [3]. The mutation alters a glycine residue that has been conserved across vertebrates. The mutation is homozygous in affected horses. Screening of control Quarter horses indicates a $3.5 \%$ carrier frequency [3]. CypB is a peptidyl prolyl cis-trans-isomerase (PPIase) found in the endoplasmic reticulum (ER) [4,5]. CypB plays a significant role in the triple helix folding of collagen [6]. It catalyzes the slow cis-trans isomerization of multiple proline residues in the collagen chain. Severe osteogenesis imperfecta (brittle bone disease) was observed in patients with mutations in СypB [7] or in CypB-deficient mice [8]. The more prevalent autosomal dominant forms of osteogenesis imperfecta are caused by primary defects in type I collagen, whereas autosomal recessive forms are caused by deficiency of proteins like CypB which interact with type I procollagen during post-translational modification and/or folding [9]. СypB forms a complex with prolyl 3-hydroxylase and cartilage-associated protein [10]. 

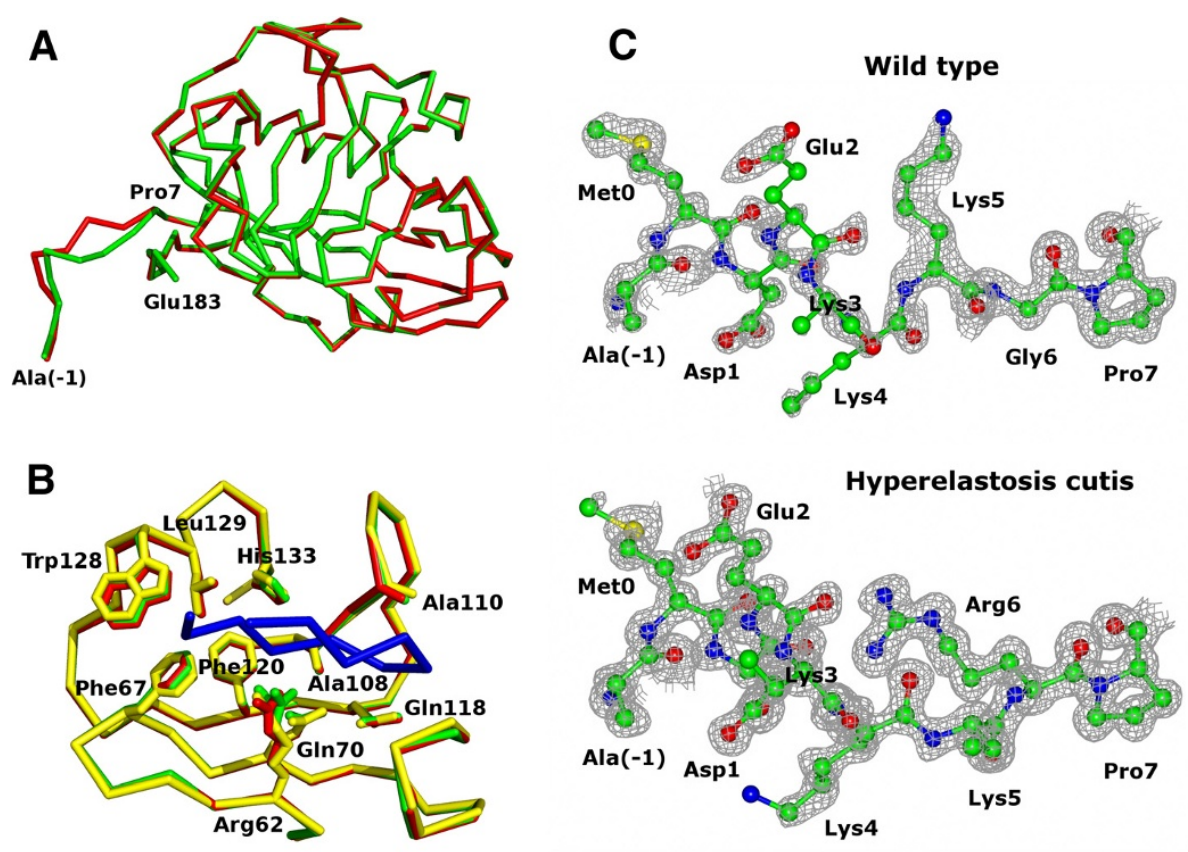

D

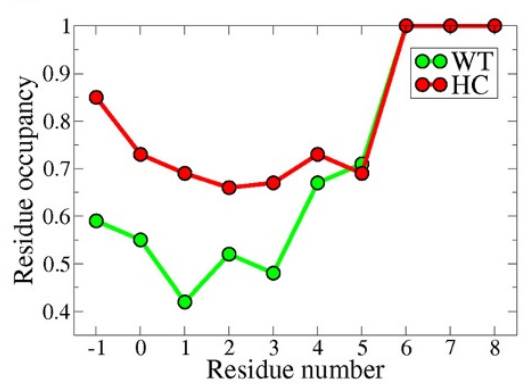

E

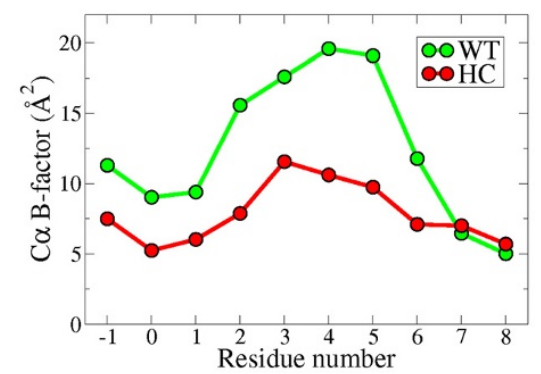

$\mathbf{F}$
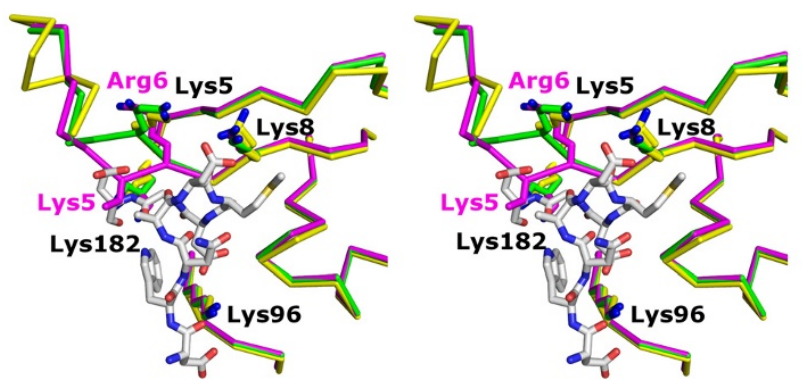

Figure 1 Crystal structures of wild-type and HC CypB. A. Ca ribbon presentation of horse wild-type (green) and HC (red) CypB. Chain displacement is observed within the N-terminal tail (residues from Ala(-1) to Pro7) B. Superimposed active site of horse wild-type (green), HC (red) and human (yellow; PDB: 1CYN [18]) CypB. Cyclosporin A is shown as ribbon (blue), which is a part of the complex with human CypB. Sidechains of residues that contribute to the binding of cyclosporin A are shown as sticks. C. Electron density maps for wild-type and HC CypB molecules calculated using 2Fo-Fc coefficients contoured at 1.00 and shown in gray. D. Refined residue occupancy values of the $\mathrm{N}$-terminal sequence for the wild-type (green) and HC (red) CypB models. E. Refined Ca B factors of the N-termini for the wild-type (green) and HC (red) CypB models. F. Stereo pair of superimposed structures of horse wild-type CypB (green), HC CypB (magenta) and the human CypB (yellow) complexed with the P-domain of calmegin [14] (white). 
The complex plays an important role in collagen biosynthesis [11]. Like other ER chaperones and foldases, CypB is involved in formation of transient multi-protein complexes that facilitate individual protein activities on nascent chains [12]. The binding partners of CypB are PDI (protein disulfide-isomerase), HSP47 (47kDa heat shock protein), BiP (binding immunoglobulin protein), GRp94 (94 kDa glucose-regulated protein), ERp72 (ER resident protein 72 ), ERp5 (ER protein 5), calreticulin, calnexin [13].

Here, we present the crystal structures of recombinantly produced wild-type and $\mathrm{HC}$-mutant $\mathrm{CypB}$ from the horse. The only difference is within the N-terminal labile end that plays a role in complex formation with other ER-resident chaperones and foldases.

\section{Results}

\section{Overall structure}

Wild-type and HC CypB were crystallized using the same crystallization conditions in space group $\mathrm{C} 2$ with one molecule per asymmetric unit. Therefore, all differences observed in the atomic models are attributable to the $\mathrm{HC}$ mutation (Gly6->Arg). Human and horse CypB share $96 \%$ sequence identity and the crystal structures are near identical ( $\mathrm{rmsd}<0.46 \AA$ for 178 equivalent $\mathrm{C} \alpha$ positions, human CypB PDB: $3 \mathrm{ICH}$ [14]). The HC СypB molecule demonstrates the same overall structure with the exception for the very short $\mathrm{N}$-terminal region that precedes the mutation site (Figure $1 \mathrm{~A})(\mathrm{rmsd}=1.31 \AA$ for 8 equivalent $C \alpha$ positions). The catalytic site remains undisturbed in the $\mathrm{HC}$ CypB molecule (Figure 1B).

\section{$\mathrm{N}$-terminal region}

Strong electron density is observed for all N-terminal residues of HC CypB (Figure 1C). While slightly weaker, electron density in this region was sufficient to unambiguously model the wild-type structure. In a previously reported high-resolution structure of human СypB the first three residues are disordered [14]. The presence of two recombinantly added amino acids (Ala-Met) preceding the natural sequence in our constructs of both $\mathrm{HC}$ and normal CypB accounts for a partial stabilization of the N-terminal segment via charge/polar contacts of Ala $(-1)$ with His133 of a neighbor molecule (a zinc ion coordinates this interaction) and hydrophobic environment for Met0 formed by residues Met68, Ala108 and Phe120 of the adjacent molecule. The conformations of $\mathrm{N}$-terminal sequences are only partially populated as revealed by the difference map, therefore variable occupancies were allowed for residues ranging from $\mathrm{Ala}(-1)$ to Lys(5) (Figure 1D). Moreover, weak difference densities from Fo-Fc maps in the vicinity of $\mathrm{Ala}(-1)$ to Lys 5 suggest that the structure might adopt alternate conformations in this region, but only one conformation could be modeled. The $\mathrm{N}$-terminal sequences appear to be the most flexible, with refined B-factors higher than average (Figure 1E). The Gly6->Arg mutation in HC CypB appears to endow greater stability in the $\mathrm{N}$-terminal region, as residues $\mathrm{Ala}(-1)$ to $\mathrm{Arg} 6$ in the $\mathrm{HC}$ mutant structure have higher occupancy values for residues ( $\sim 0.7$ vs. $\sim 0.5$ for wild-type) and significantly lower refined B-factors (5-12 $\AA^{2}$ vs. 9-20 $\AA^{2}$ for wild-type). Thus, the Gly6->Arg mutation causes significant change in the structure (up to $4.2 \AA$ shift for the backbone at residue Lys5), with the wild-type side chain density of Lys5 being partially occupied by HC Arg6, and the flexibility of the N-terminal region sharply reduced.

\section{P-domain interaction interface}

The recently reported crystal structure of human CypB complexed with the proline-rich P-domain of calmegin reveals that the binding involves the $\mathrm{N}$-terminal region of cyclophilin and the surfaces of $\mathrm{CypB}$ and $\mathrm{P}$-domain are complementary [14]. That binding site is on the opposite side of CypB than the peptidyl prolyl cis-trans-isomerase activity site. The N-terminal region forms part of the Pdomain binding interface, contributing several hydrogen bonds. In $\mathrm{HC}$ CypB, the binding interface is altered: CypB overlaps with the P-domain, when superimposed, such that it could adversely affect binding (Figure 1F).

\section{Discussion}

Our structures suggest a molecular rationale for the disease phenotype of the $\mathrm{HC}$ mutation in CypB of the American quarter horse. Evidence is accumulating that the $\mathrm{N}$-terminal region of СурВ interacts not only with calreticulin, but also with ER chaperones and foldases. Indeed, we have recently shown an altered interaction between $\mathrm{HC}$ CypB with the P-domain of calreticulin and loss of complex formation with lysyl hydroxylase 1 [15]. Interactions of CypB with ERp72 and GRp94 also require the $\mathrm{N}$-terminal tail as confirmed by NMR experiments with the strongest chemical shifts observed for residues Lys3, Lys4, Lys5, Lys8 [13]. The same mode of СypB binding might be valid for BiP, PDI, ERp5 and calreticulin, where a polyacidic sequence similar to the one located in ERp72 was recognized [13]. The Gly6->Arg mutation in HC CypB has two consequences; first, it adds an additional positive charge to the polybasic stretch, which is involved in the binding of the polyacidic region in calreticulin and thus can enhance the affinity, second, it significantly distorts the geometry and flexibility of the $\mathrm{N}$-terminal tail and thus can slow down, limit or even eliminate binding-competent conformations. As seen in Figure 1F a conformation of the HC CypB N-terminal tail is likely incompatible with $\mathrm{P}$ domain interactions. Nevertheless, $\mathrm{HC}$ CypB binds the $\mathrm{P}$-domain of calreticulin, but shows aberrant kinetics 
when compared with the wild-type; extra slow phases are observed for both association and dissociation [15]. The two consequences of the mutation in $\mathrm{HC}$ CypB have a synergistic effect on the overall $\mathrm{P}$-domain binding. Binding of lysyl hydroxylase 1 to $\mathrm{HC} \mathrm{CypB} \mathrm{is} \mathrm{fully}$ abolished [15], which establishes a more important role of the N-terminal tail and a stronger requirement for the $\mathrm{N}$-terminal tail flexibility. Three lysine residues preceding the mutated glycine residue can be involved in $\mathrm{N}^{\varepsilon}$ acetylation, which was recently reported for CypB [16]. The mutation might affect acetylation and this could also cause changes in the repertoire and mode of interactions with other proteins.

\section{Conclusion}

Hyperelastosis cutis is a connective tissue disorder caused by the Gly6->Arg mutation in cyclophilin B. The high resolution structures of the recombinant wild-type and mutated horse cyclophilin B provide evidence that the active enzymatic site is undisturbed and suggesting that the disease phenotype is caused by changes in the structure and flexibility of the $\mathrm{N}$-terminal tail, altering interactions with other ER-resident chaperones and foldases.

\section{Methods}

\section{Expression and purification of wild-type and HC} cyclophilin B

The wild-type and $\mathrm{HC}$ CypB were recombinantly produced in bacteria and purified as described [15].

\section{Crystallization and datacollection}

The purified recombinant wild-type and $\mathrm{HC}$ horse CypB were dialyzed against $10 \mathrm{mM}$ Tris/ $\mathrm{HCl}, \mathrm{pH} 7.2$, and concentrated to about $6 \mathrm{mg} / \mathrm{ml}$ using an Amicon spin concentrator. The proteins were crystallized at room temperature using the hanging drop vapor diffusion method. For crystallization, $1 \mu \mathrm{l}$ of the peptide solution was mixed with $1 \mu \mathrm{l}$ of the reservoir solution containing $0.1 \mathrm{M}$ MES, $10 \mathrm{mM} \mathrm{ZnCl}_{2}, 10 \%$ glycerol and $28 \%$ polyethylene glycol monomethyl ether $550, \mathrm{pH}$ 6.5. The crystals of hexagonal shape appeared within 2-5 days. The crystals were directly frozen in liquid nitrogen since the

Table 1 Summary of data collection and refinement statistics

\begin{tabular}{|c|c|c|}
\hline & Wild-type CypB (4FRU) & HC СурB (4FRV) \\
\hline \multicolumn{3}{|l|}{ Data collection } \\
\hline Space group & $\mathrm{C} 2$ & C2 \\
\hline$a, b, c(\AA)$ & $64.9,44.1,60.6$ & $64.8,44.2,60.1$ \\
\hline$a, \beta, \gamma\left(^{\circ}\right)$ & $90,95.2,90$ & $90,95.5,90$ \\
\hline Resolution ${ }^{a}(\AA)$ & $16.4-1.1(1.16-1.10)$ & 16.3-1.1 (1.16-1.10) \\
\hline Measured reflections ${ }^{\mathrm{a}}$ & $208,598(10,718)$ & $206,447(10,544)$ \\
\hline Unique reflections $^{a}$ & $62,397(5,316)$ & $61,849(5,304)$ \\
\hline Redundancy ${ }^{a}$ & $3.3(2.0)$ & $3.3(2.0)$ \\
\hline Completeness $^{a}(\%)$ & $90.4(53.4)$ & $90.2(53.4)$ \\
\hline Matthews coefficient $\left(\AA^{3} \mathrm{Da}^{-1}\right)$ & 2.1 & 2.1 \\
\hline Solvent fraction (\%) & 41.8 & 41.1 \\
\hline$|/ \sigma|^{a}$ & $9.8(3.9)$ & $12.4(4.2)$ \\
\hline Wilson plot B-factor $\left(\AA^{2}\right)$ & 4.2 & 4.4 \\
\hline $\mathrm{R}_{\text {merge }}^{\mathrm{a}}(\%)$ & $7.8(27.2)$ & $5.2(24.2)$ \\
\hline \multicolumn{3}{|l|}{ Refinement } \\
\hline R-factor (\%) & 11.6 & 11.9 \\
\hline$R_{\text {free }}(\%)$ & 13.9 & 13.8 \\
\hline Protein/solvent atoms ${ }^{b}$ & $1472 / 300$ & $1481 / 293$ \\
\hline Rmsd. of bond lengths $(\AA)$ & 0.007 & 0.007 \\
\hline Rmsd. of bond angles $\left(^{\circ}\right)$ & 1.30 & 1.29 \\
\hline Average $B$ value ${ }^{c}\left(\AA^{2}\right)$ & $10.0(7.8)$ & $10.2(7.2)$ \\
\hline Ramachandran favored/allowed/outliers (\%) & $97.8 / 2.2 / 0$ & $97.8 / 2.2 / 0$ \\
\hline MolProbity score [22] & $0.64\left(100^{\text {th }}\right.$ percentile $)$ & 0.90 (99 $9^{\text {th }}$ percentile) \\
\hline
\end{tabular}

${ }^{a}$ Values in parentheses are for the highest resolution shell.

${ }^{b}$ Excluding hydrogen atoms.

${ }^{c}$ Values in parentheses are for macromolecule atoms. 
10\% glycerol / 28\% PEG MME 550 sufficed as the cryoprotectant condition.

Data collection was performed using crystals flash-frozen to $100 \mathrm{~K}$ on the "NOIR-1" detector system at the Molecular Biology Consortium Beamline 4.2.2 of the Advanced Light Source, Lawrence Berkeley National Laboratory.

\section{Crystal structure determination}

The images collected were indexed, integrated and scaled using MOSFLM and SCALA from the CCP4 suite [17]. The AMORE program was used within the CCP4 suite [17] to find initial molecular replacement solutions. A crystal structure of human cyclophilin B (PDB: 1CYN) [18] was used as a search model. A single outstanding solution was generated by AMORE. Iterative cycles of model correction and refinement were performed using COOT [19] and PHENIX [20], respectively. Hydrogens were added and refined in riding positions. B factors of all atoms except hydrogens were refined anisotropically. Seven alternative side chain conformations were built for the models of both the wild-type and HC CypB. Two zinc ions were found to be involved in crystal contacts. Two short fragments of polyethylene glycol monomethyl ether 550 were fitted near each model. The quality of the models was assessed with program MolProbity (http://molprobity.biochem.duke.edu/). Data collection and refinement statistics are summarized in Table 1. Figures were generated with programs PyMOL (http://www. pymol.org) and CCP4MG [21].

\section{Availability of supporting data}

The atomic coordinates and structure factors have been deposited with the protein Data Bank (PDB: 4FRU for wild-type and 4FRV for $\mathrm{HC}$ CypB).

\section{Abbreviations \\ HC: Hyperelastosis cutis; CypB: Cyclophilin B; HERDA: Hereditary equine regional dermal asthenia; ER: Endoplasmic reticulum; PDI: Protein disulfide- isomerase; HSP47: 47kDa heat shock protein; BiP: Binding immunoglobulin protein; GRp94: 94 kDa glucose-regulated protein; ERp72: ER resident protein 72; ERp5: ER protein 5.}

\section{Competing interests}

The authors declare that they have no competing interests.

\section{Authors' contributions}

SPB, YI, and HPB conceived the study. YI purified and crystallized the proteins. JN collected the diffraction data. SPB solved the crystal structures, analyzed the data, and wrote the manuscript. TL and MSC helped with the diffraction data collection, analyzed the data and edited the manuscript. All authors read and approved the final manuscript.

\section{Acknowledgments}

The authors thank Nena Winand for the horse DNA and encouragement for this project. HPB is supported by the grant from Shriners Hospital for Children; TFL is supported by the Interactions at the Microbe-Host Interface training grant from the National Institutes of Health. (T32Al007472).

\section{Author details}

${ }^{1}$ Research Department, Shriners Hospital for Children, Portland, OR 97239, USA. 'Department of Biochemistry and Molecular Biology, Oregon Health \& Science University, Portland, OR 97239, USA. ${ }^{3}$ Molecular Biology Consortium, Advanced Light Source Beamline 4.2.2, Lawrence Berkeley National Laboratory, 1 Cyclotron Road, Berkeley, CA 94720, USA.

Received: 20 September 2012 Accepted: 1 November 2012 Published: 8 November 2012

\section{References}

1. White SD, Affolter VK, Bannasch DL, Schultheiss PC, Hamar DW, Chapman PL, Naydan D, Spier SJ, Rosychuk RA, Rees C, et al: Hereditary equine regional dermal asthenia ("hyperelastosis cutis") in 50 horses: clinical, histological, immunohistological and ultrastructural findings. Vet Dermatol 2004, 15(4):207-217.

2. Mao JR, Bristow J: The Ehlers-Danlos syndrome: on beyond collagens. J Clin Invest 2001, 107(9):1063-1069.

3. Tryon RC, White SD, Bannasch DL: Homozygosity mapping approach identifies a missense mutation in equine cyclophilin B (PPIB) associated with HERDA in the American Quarter Horse. Genomics 2007, 90(1):93-102.

4. Price ER, Zydowsky LD, Jin MJ, Baker CH, McKeon FD, Walsh CT: Human cyclophilin B: a second cyclophilin gene encodes a peptidyl-prolyl isomerase with a signal sequence. Proc Natl Acad Sci USA 1991, 88(5):1903-1907.

5. Hasel KW, Glass JR, Godbout M, Sutcliffe JG: An endoplasmic reticulumspecific cyclophilin. Mol Cell Biol 1991, 11(7):3484-3491.

6. Steinmann B, Bruckner P, Superti-Furga A: Cyclosporin A slows collagen triple-helix formation in vivo: indirect evidence for a physiologic role of peptidyl-prolyl cis-trans-isomerase. J Biol Chem 1991, 266(2):1299-1303.

7. van Dijk FS, Nesbitt IM, Zwikstra EH, Nikkels PG, Piersma SR, Fratantoni SA, Jimenez CR, Huizer M, Morsman AC, Cobben JM, et al: PPIB mutations cause severe osteogenesis imperfecta. Am J Hum Genet 2009, 85(4):521-527.

8. Choi JW, Sutor SL, Lindquist L, Evans GL, Madden BJ, Bergen HR 3rd, Hefferan TE, Yaszemski MJ, Bram RJ: Severe osteogenesis imperfecta in cyclophilin B-deficient mice. PLoS Genet 2009, 5(12):e1000750.

9. Forlino A, Cabral WA, Barnes AM, Marini JC: New perspectives on osteogenesis imperfecta. Nat Rev Endocrinol 2011, 7(9):540-557.

10. Vranka JA, Sakai LY, Bächinger HP: Prolyl 3-hydroxylase 1, enzyme characterization and identification of a novel family of enzymes. J Biol Chem 2004, 279(22):23615-23621.

11. Ishikawa Y, Wirz J, Vranka JA, Nagata K, Bächinger HP: Biochemical characterization of the prolyl 3-hydroxylase 1.cartilage-associated protein.cyclophilin B complex. J Biol Chem 2009, 284(26):17641-17647.

12. Braakman I, Bulleid NJ: Protein folding and modification in the mammalian endoplasmic reticulum. Annu Rev Biochem 2011, 80:71-99.

13. Jansen G, Maattanen P, Denisov AY, Scarffe L, Schade B, Balghi H, Dejgaard K, Chen LY, Muller WJ, Gehring K, et al: An interaction map of ER chaperones and foldases. Mol Cell Proteomics 2012, 11(9):710-723.

14. Kozlov G, Bastos-Aristizabal S, Maattanen P, Rosenauer A, Zheng F, Killikelly A, Trempe JF, Thomas DY, Gehring K: Structural basis of cyclophilin B binding by the calnexin/calreticulin P-domain. J Biol Chem 2010, 285(46):35551-35557.

15. Ishikawa Y, Vranka JA, Boudko SP, Pokidysheva E, Mizuno K, Zientek K, Keene DR, Rashmir-Raven AM, Nagata K, Winand NJ, et al: The mutation in cyclophilin B that causes hyperelastosis cutis in the American Quarter Horse does not affect peptidyl-prolyl cis-trans isomerase activity, but shows altered cyclophilin B-protein interactions and affects collagen folding. J Biol Chem 2012, 287:22253-22265.

16. Pehar M, Lehnus M, Karst A, Puglielli L: Proteomic assessment shows that many endoplasmic reticulum (ER)-resident proteins are targeted by $\mathrm{N}$ (epsilon)-lysine acetylation in the lumen of the organelle and predicts broad biological impact. J Biol Chem 2012, 287(27):22436-22440.

17. Collaborative Computational Project N: The CCP4 suite: programs for protein crystallography. Acta Crystallogr D: Biol Crystallogr 1994, 50(Pt 5):760-763. 
18. Mikol V, Kallen J, Walkinshaw MD: X-ray structure of a cyclophilin B/ cyclosporin complex: comparison with cyclophilin A and delineation of its calcineurin-binding domain. Proc Natl Acad Sci USA 1994, 91(11):5183-5186.

19. Emsley P, Lohkamp B, Scott WG, Cowtan K: Features and development of Coot. Acta Crystallogr D: Biol Crystallogr 2010, 66(Pt 4):486-501.

20. Adams PD, Afonine PV, Bunkoczi G, Chen VB, Davis IW, Echols N, Headd JJ, Hung LW, Kapral GJ, Grosse-Kunstleve RW, et al: PHENIX: a comprehensive Python-based system for macromolecular structure solution. Acta Crystallogr D: Biol Crystallogr 2010, 66(Pt 2):213-221.

21. McNicholas S, Potterton E, Wilson KS, Noble ME: Presenting your structures: the CCP4mg molecular-graphics software. Acta Crystallogr D: Biol Crystallogr 2011, 67(Pt 4):386-394.

22. Chen VB, Arendall WB 3rd, Headd JJ, Keedy DA, Immormino RM, Kapral GJ, Murray LW, Richardson JS, Richardson DC: MolProbity: all-atom structure validation for macromolecular crystallography. Acta Crystallogr D Biol Crystallogr 2010, 66(Pt 1):12-21.

doi:10.1186/1756-0500-5-626

Cite this article as: Boudko et al: Crystal structures of wild-type and mutated cyclophilin B that causes hyperelastosis cutis in the American quarter horse. BMC Research Notes 2012 5:626.

\section{Submit your next manuscript to BioMed Central and take full advantage of:}

- Convenient online submission

- Thorough peer review

- No space constraints or color figure charges

- Immediate publication on acceptance

- Inclusion in PubMed, CAS, Scopus and Google Scholar

- Research which is freely available for redistribution 